Physical Sciences | Wijnand Broer

\title{
A long-awaited understanding of the Casimir torque
}

The Casimir force has been well researched by physicists for decades, but only recently has one particularly intriguing aspect of is effect been his research, Dr Wijnand Broer at the University of Chinese Academy of Sciences has built upon these recent breakthroughs to explore the processes which cause the surfaces of some specialised materials to rotate when placed close to each other. His the first updated explanations the first updated explanations
of this 'Casimir torque' in over 40 years and could soon lead to a diverse range of technological application

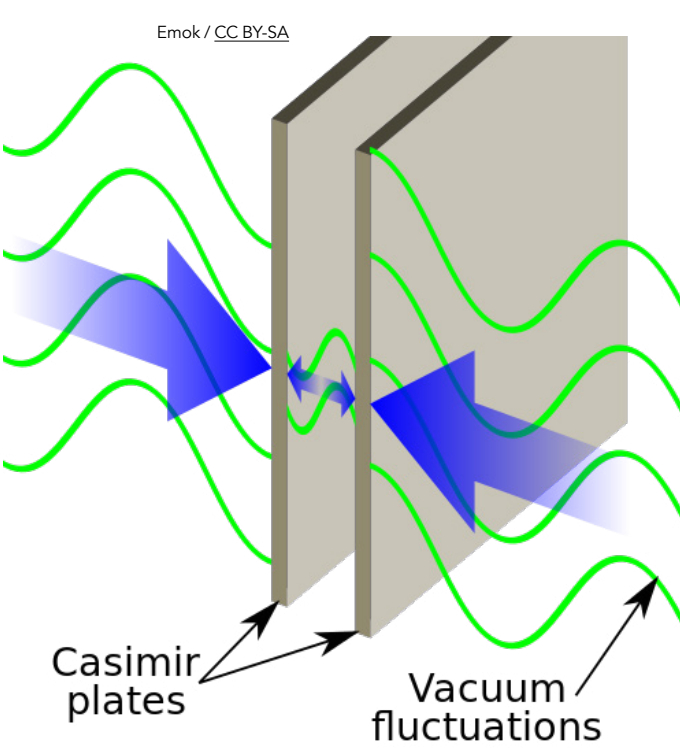

$\triangle \begin{aligned} & \text { Ithough a perfect vacuum } \\ & \text { is completely devoid of }\end{aligned}$ iny matter, the physical strange laws of quantum mech the 'fluctuations' in the vacuum can cause electromagnetic waves to emerge, seemingly out of nowhere. In 1948, Dutch physicist Hendrik. Casimir predicted that rather than being an abstract concept, these fluctuations can exert real forces on physical objects: namely, two electrically neutral, reflecting surfaces which face each other. "When such electromagnetic fields travel from on surface to another, they give rise to interaction between the surfaces: the so-called the "Casimir force , explains DrWijnand Broer, senior postdoc at the University of Chinese Academy attractive in most cases, meaning it tries to pull the surfaces together."

The effect is particularly prominent for surfaces placed just nanometres or micrometres apart. This is because the pressure exerted on them by the within such small gaps is generally smaller than that exerted by waves outside, meaning they are noticeably pushed together. Although this was seen as little more than an interesting curiosity in Casimir's time, the Casim force is now becoming increasingly relevant to modern physics as our technology becomes smaller, and as universe emerge.
Alternatively, this effect can be understood as a consequence of positions of the chal uncertainty of the These charge fluctuations give rise to temporary differences between the charge distributions of the surfaces, leading to a macroscopic force betwe them. At relatively short distances, where light speed seems infinite and the Casimir interaction simultaneous, the Casimir force is sometimes also called the van der Waals force.

"The Casimir force is studied both for practical reasons, concerning tiny machines whose components are in close proximity to each other, and fundamental reasons, including shortrange measurements of gravity, and Broer new fundamental forces", Dr (B) which give rise to the Casimir force, its effects in some more complex situations have remained most mysterious until recently.

\section{DIRECTIONAL DEPENDENCE} Light will always slow down when it travels through any medium other than a vacuum. The degree to which quantified by a value named the the speed of ligh - or the ratio between the speed of light inside the material to its speed in a vacuum.

As first shown by the Soviet physicist Evgeny Lifshitz, the refractive indices characteristics of the Casimir force when placed opposite each other in force, one has to understand how the electromagnetic field propagates hrough the materials by solving

Maxwell's equations.

For conventional materials like window glass, the value of the refractive index remains independent of the direction in which it travels through the material. However, it isn't so simple to define the refractive indices of some materials with more intricate molecular structures. 'birefringent' materials, for example, light waves travelling in different different degrees.

"Some materials have multiple fractive indices, since the speed propagates in", Dr Broer describes. "The simplest example of such an 'anisotropic' substance is a birefringent material, with two refractive indices."

Due to these differences in speed, virtual light waves travelling in different directions will impart different forces on different parts of birefringent material surfaces. This results in a "torque which forces both objects to rotate. As Dr Broer continues, "the Casimin interaction between two of these anisotropic surfaces gives rise to a torque that is called the Carinir onc." This is called the Carimir toque." These the Casimir force, and are far more complex to describe the a simple ttraction between two reflecting surfaces. All the same, the Casimir

\section{RECREATING THE CASIMIR}

TOROUE IN THE LAB

In 1978, a Russian physicist named Yur Barash predicted the Casimir torque through theoretical calculations. He did this by considering how two oppositefacing anisotropic surfaces would need to rotate to reach a position for minimum energy. Yet in the years following his discovery, physicists had no success in demonstrating Barash's remained until very recently. "The

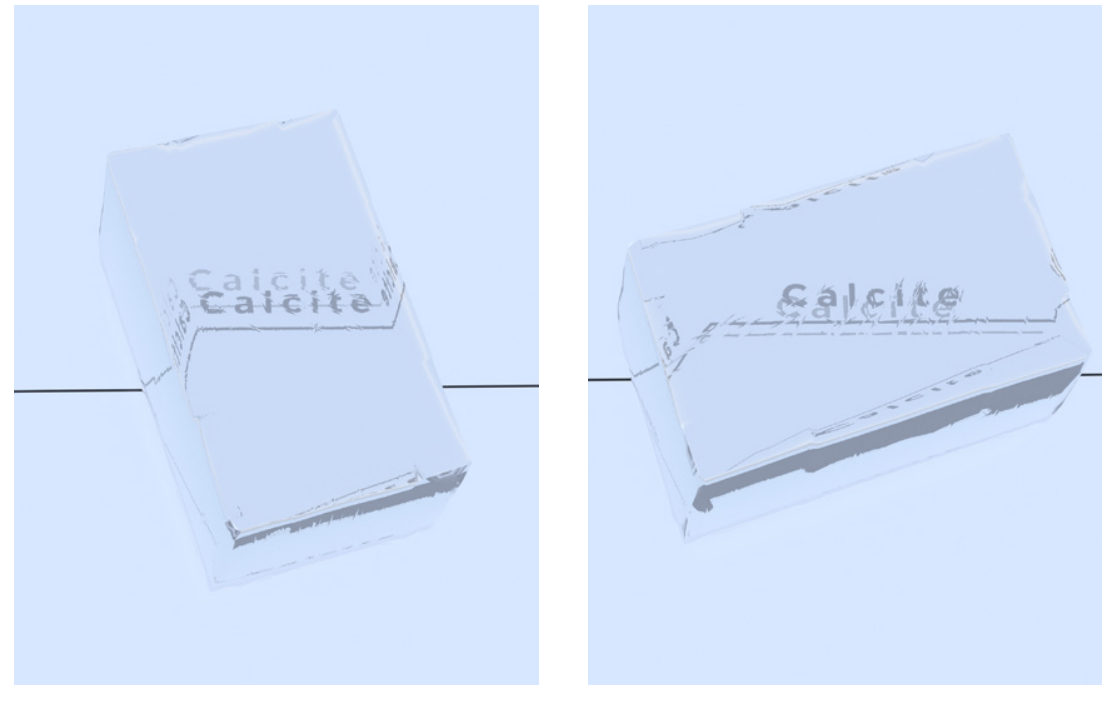

When a birefringent crystal is rotated, one version of the text changes with its orientation whereas the
other one doesn't. The former is called the 'extraordinary' mode and the latter is the 'ordinary' mode.

first exact expression for birefringent 40 years ago, but it first experimenta observation was only accomplished in 2018", Dr Broer recounts.

In this study, a team at the University of Maryland quantified the Casimir torque for the first time using a liquid crystal - a material which flows like a liquid, but whose molecules remain oriented in crystal-like structures which can be

Institute in Ljubljana, Slovenia, revealed that similar dynamics can arise due to similar effect to the Casimir force,

They arise as the motions of electrons within individual molecules induce small, short-term charges within the particles, creating weak forces between different materials.

The researchers observed the effect different layers: some isotropic, meaning their refractive indices

Virtual light waves travelling in different directions will impart different forces on birefringent material surfaces.

This results in a 'torque' which forces both objects to rotate.

liquid crystal is placed close to a solid birefringent substrate, the orientations of its constituent molecules twisted

The team then repeated the experiment using several different materials, allowing them to control the strength and

direction of the torque, its dependence .

\section{PREDICTING VAN DER}

\section{WAALS TOROUES}

Through a separate study published

in 2016, researchers at the J. Stefan remained the same no matter the light direction, and some anisotropic to different degrees. Although this configuration was more complicated the one studied by Barash, (a) being infinite. They also adjusted the hickness of the layers and arranged the slabs at different angles. Like the University of Maryland team, they demonstrated a clear, tuneable torque with their setup, providing Barash's calculations. 


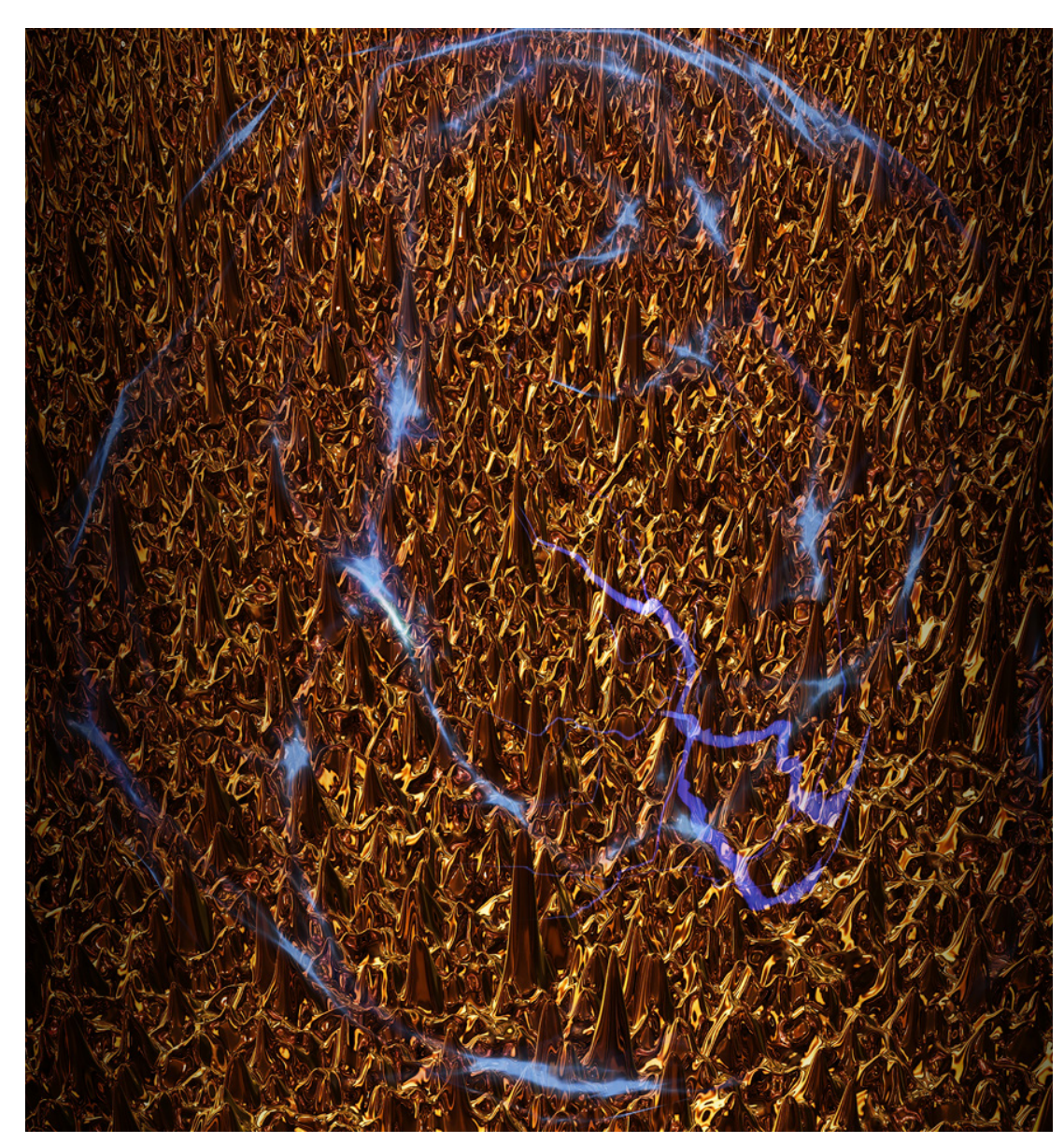

Fluctuating charges and currents in a rough gold surface: such fluctua
and quantum mechanical in nature, give rise to Casimir interactions.

Such discoveries could bring about a wealth of technological applications, including nanoscale devices which can be actively controlled by a user.

\section{A NEW UNDERSTANDING}

\section{OF THE CASIMIR TOROUE}

Despite the numerous successes of

issue remained: theoretical physicists

in past decades have struggled to

independently verify Barash's result

when starting their calculations of

the Casimir torque from scratch. This was a problem since for any theory in physics, such independent methods are critically important for confirming that the original theory was robust in the first place. "Reproducing Barash's expression theoretically and independently has proved difficult over the years", Dr Broer explains. "Some results have appeared, but so far, it has remained unclear whet'ser
consistent with Barash's." a transparent birefringent crystal the background to be split into two arions. Depending on its totation, such In the 'ordinary' mode, the calcite. behaves like regular isotropic glass, an the version of the background which travels through the crystal retains its orientation. If the calcite is rotated, however, it will be in an 'extraordinary' mode, meaning the version which passes through will be rotated with respect to the original background.

"We have derived a result for the Casimir torque using a mathematica method that determines how light is split up as it propagates through a birefringent medium", Dr Broer describes. "We have managed to show sest splitting up this large, complicated Furthermore, the team's approach can be generalised to incorporate a wide range of anisotropic materials with different thicknesses and compositions, opening up exciting new opportunities for research.

\section{APPLICATIONS AND MORE} COMPLEX SITUATIONS

Dr Broer now hopes that by applying

his team's theoretical approaches,

researchers in future studies will pus

our understanding of the physics

underlying the Casimir torque even further. With this, we hope to have shed some light on these complicated weractions. Moreover, he method we used can be generalised to more complicated sichations such as biaxial indices", he concludes. from both the J. Stefan Institute and and his colleagues aimed in their latest research to solve this issue starting from Maxwell's equations - the universal mathematics describing the behaviours of all electromagnetic waves. Throug their innovative calculations, the researchers could finally reproduce Barash's original results, allowing them to make highly accurate theoretical predictions of the Casimir torque in different situations.

One situation which the theories can describe in this way can be seen wh such as calcite is placed on top of
Such discoveries could bring about a wealth of technological applications, including nanoscale devices which can be actively controlled by a user. These would be particularly useful for realising 'microelectromechanical' systems - intelligent devices in which tiny mechanisms and sensors are carved into silicon substrates and wired to electronic components. Ultimately, his team's findings represent a significant breakthrough in our understanding of the Casimir torque, and provide long-awaited stumped many physists for decades.

\section{(3) Behind the Research}

W E: wijnand@ucas.ac.cn T: $\quad$ +86 15210391401

E: wijnand@ucas.ac.cn T: +8615210391401
W: https:///www.researchgate.net/profile/Wijnand_Broer

\section{Research Objectives}

Dr Broer studies the phenomenon of the Casimir torque and explains how it relates to light splitting up as it

\section{Detail}

Wijnand Broer

Institute of Physics

University of Chinese Academy of Sciences 100190 Beijing

China

Bio

DrWijnand Broer obtained his PhD in 2014 at the University of Groningen, the Netherlands. After postdoc fellowships in Germany and Singapore, he is presently a senior postdoc at the Universt

Funding

- Nanyang Technological University, Singapore

University of Chinese Academy of Sciences

Collaborators

Bing-Sui Lu Assistant professor, Nanyang

Technological University, Singapore

Rudolf Podgornik, Professor, Chinese Academy of
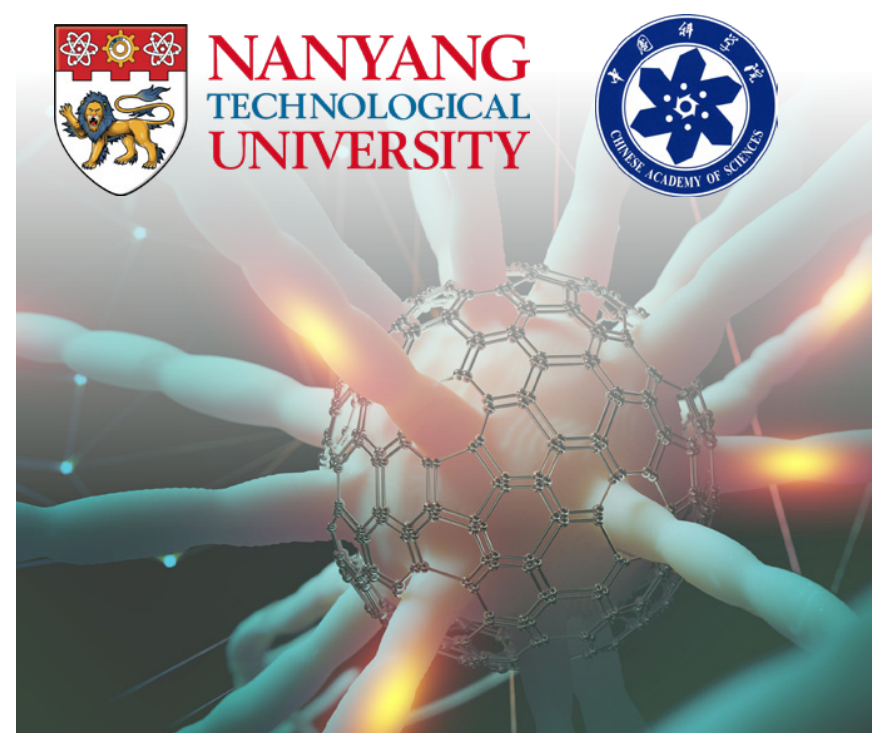

\section{References}

Barash, Y.S. (1978). Moment of van der Waals forces between anisotropic bodies. Radiophys. Quant. Electronics, 21, 1138 .

Broer, W., Liow, J.Y.H., and Lu, B.S. (2019). Maxwell eigenmode approach to the Casimir-Lifshitz torque.

Broer, W., Palasantzas, G., Knoester, J., and Svetovoy, V.B. (2013). Significance of the Casimir force and surface roughness for actuation dynamics of MEMS. Physical Review B, 87(12), 125413

Broer, W., Waalkens, H., Svetovoy, V.B., Knoester, J., and Palasantzas, G. (2015). Nonlinear actuation dynamics of driven Casimir oscillators with rough surfaces. Physical Review Applied, 4(5), 054016.

Casimir, H.B.G. (1948). On the Attraction Between Two Perfectly Conducting Plates. Proc. K. Ned. Akad. Wet., Ser. $B, 51,793$.

Lifshitz, E.M. (1956). The Theory of Molecular Attractive Forces between Solids. Sov. Phys. JETP, 2(1), 73

Lu, B.S., and Podgornik, R. (2016). Van der Waals torque and force between dielectrically anisotropic layered media. The Journal of Chemical Physics, 145(4), 044707.

Somers, D.A., Garrett, J.L., Palm, K.J., and Munday, J.N.

(2018). Measurement of the Casimir torque. Nature,

564(7736), 386-389.

\section{Personal Response}

Do you have plans for future studies on the Casimir torque?

I intend to continue investigating fluctuationinduced interactions in the context of applications in microelectromechanical systems and new materials. Dos materials, and magnetically anisorto, soft matter, 